\title{
Impact of depression on gait variability in Parkinson's disease
}

\author{
Nataša T Dragašević-Miškovićc ${ }^{\mathrm{a}, *}$, Vladislava Bobić ${ }^{\mathrm{b}, \mathrm{c}}$, Milutin Kostić $^{\mathrm{d}}$, Iva Stanković ${ }^{\mathrm{a}}$, \\ Saša Radovanoviće ${ }^{\mathrm{e}}$, Kosta Dimitrijević ${ }^{\mathrm{a}}$, Marina Svetel ${ }^{\mathrm{a}}$, Igor Petrovićc ${ }^{\mathrm{a}}$, Milica Đurić-Jovičić ${ }^{\mathrm{b}}$ \\ ${ }^{a}$ Neurology Clinic, Clinical Center Serbia, School of Medicine, University of Belgrade; dr Subotića 6a, Belgrade, Serbia \\ ${ }^{\mathrm{b}}$ Innovation Center, School of Electrical Engineering in Belgrade, Bulevar kralja Aleksandra 73, Belgrade, Serbia \\ ${ }^{\mathrm{c}}$ School of Electrical Engineering, University of Belgrade, Bulevar kralja Aleksandra 73, Belgrade, Serbia \\ ${ }^{\mathrm{d}}$ Institute of Mental Health, Palmotićeva 37, Belgrade, Serbia \\ ${ }^{\mathrm{e}}$ Institute for Medical Research, University of Belgrade, dr Subotica 4, Belgrade, Serbia
}

\section{A R T I C L E I N F O}

\section{Keywords:}

Parkinson's disease

Depression

Gait analysis

Gait variability

Dual-task paradigm

\begin{abstract}
A B S T R A C T
Objective: The goal of this study was to analyze how depression associated with Parkinson's disease (PD) affected gait variability in these patients using a dual-task paradigm. Additionally, the dependency of the executive functions and the impact of depression on gait variability were analyzed.

Patients and Methods: Three subject groups were included: patients with PD, but no depression (PD-NonDep; 14 patients), patients with both PD and depression (PD-Dep; 16 patients) and healthy controls (HC; 15 subjects). Gait was recorded using the wireless sensors. The participants walked under four conditions: single-task, motor dual- task, cognitive dual-task, and combined dual-task. Variability of stride length, stride duration, and swing time was calculated and analyzed using the statistical methods.

Results: Variability of stride duration and stride length were not significantly different between PD-Dep and PDNonDep patients. The linear mixed model showed that swing time variability was statistically significantly higher in PD-Dep patients compared to controls $(\mathrm{p}=0.001)$. Hamilton Disease Rating Scale scores were significantly correlated with the swing time variability $(\mathrm{p}=0.01)$. Variability of all three parameters of gait was significantly higher while performing combined or cognitive task and this effect was more pronounced in PD-Dep group of patients.

Conclusions: Depression in PD was associated with swing time variability, and this effect was more prominent while performing a dual-task.

Significance: Diagnosing and treating depression might be important for gait improvement and fall reduction in PD patients.
\end{abstract}

\section{Introduction}

Gait disturbance is one of the particularly incapacitating signs of Parkinson's disease (PD). It is characterized by slowness, variability, and postural instability, related to impaired spinal and supraspinal locomotor networks (premotor cortical, motor cortical, basal ganglia, cerebellar, and brain stem structures). Peterson and Horak suggested that slow gait in PD might be related to a dysfunction of the basal gangliathalamocortical loop, while gait variability appeared when a shift from automatic to voluntary control of walking occurred [1]. Gait has been increasingly understood as a complex "higher-order" form of motor behavior, with prominent influences of mental processes (executive function, attention, a judgement of external/internal cues) [2]. Such impact is particularly evident under the dual-task (DT) conditions, which changes gait parameters, including stride-to-stride variability, both in healthy individuals and in PD patients [2-5].

Depression is a frequent and significant non-motor symptom of PD, with a prevalence of approximately $35 \%$ [6]. It has a modulatory effect on cognitive functions and possibly a shared pathophysiological basis with disturbances in the executive functions [7]. It has been demonstrated that unipolar depression by itself also influenced gait parameters, primarily speed and time spent with both feet on the ground $[8,9]$. Depression is an independent predictor of gait speed under single and dual-task (DT) conditions in mild to moderately severe PD [5].

Keeping in mind the interplay among cognition, gait, and depression, as well as the results of studies demonstrating the influence of

\footnotetext{
* Corresponding author at: Neurology Clinic, Clinical Center Serbia, School of Medicine, University of Belgrade, Serbia.

E-mail address: ntdragasevic@gmail.com (N.T. Dragašević-Mišković).
} 
depression on gait parameters, we wanted to investigate whether depression associated with PD affected gait variability in these patients. We have hypothesized that: 1) PD patients with major depression (PDDep) had more expressed gait variability compared to PD patients without depression (PD-NonDep), measuring these possible changes by using DT paradigm; and 2) the impact of depression on gait variability in PD patients might be, at least partially, dependent on executive functions.

\section{Materials and methods}

\subsection{Subjects}

The study included 30 consecutive, non-demented in-patients with PD, diagnosed by a specialist for movement disorders (ND, MS, or IP) according to the UK PD Society Brain Bank criteria [10], at the Neurology Clinic, Clinical Center of Serbia, Belgrade.

PD-NonDep and PD-Dep patients were included, while those with dysthymia were excluded from the study. The initial version of the Unified Parkinson's Disease Rating Scale (UPDRS) score was used to assess parkinsonian signs [11].

All patients were interviewed by a psychiatrist (MK) to diagnose major depression (MD). The Structured Clinical Interview for DSM-V Axis I Disorders (SCID-1) was used as a diagnostic exam to determine DSM-V Axis I disorders [12]. Furthermore, all patients were assessed with the Hamilton Depression Rating Scale (HDRS) [13]. The Frontal Assessment Battery (FAB) [14], Addenbrooke's Cognitive Examination-Revised scale (ACE-R) (total score) [15], Digit Span Backward test [16] and Stroop Color and Word test (Stroop interference score) [17] were used to evaluate executive functions. Tinetti Balance Score (TBS) was also assessed for all patients. Cognitive functions were tested using Mini-Mental State Examination scale (MMSE) [18]. Levodopa equivalent dose (LED) was $875.7 \pm 485.3 \mathrm{mg}$.

Exclusion criteria were: 1) significant memory impairment and MMSE $<26$ or patients who fulfilled criteria for the diagnosis of dementia; 2) independent gait without aids was not possible; 3) additional vascular, rheumatoid, and psychiatric comorbidities that might contribute to gait difficulties; and 4) neuroleptic treatment.

The control group (HC) consisted of 15 healthy, gender- and agematched subjects recruited among caregivers and healthy staff members at the Neurology Clinic.

The study was performed in accordance with the ethical standards of the Declaration of Helsinki and approved by the Ethical Committee of the School of Medicine, University of Belgrade. All the participants gave written informed consent before entering the study.

\subsection{Experiment}

The participants were asked to perform a walking task under four different conditions (no instruction was given to prioritize one task over other in DT paradigm) [19]:

1) Single-task - walking along a straight path at their usual rhythm;

2) Motor DT - same as (1) but a participant also carried a glass of water, trying not to spill it;

3) Cognitive DT - same as (1) but a participant in parallel performed mathematical task - serial subtractions of "7" from " 100 ". Subtractions were conducted loudly, and examiners took care that participants provided correct answers.

4) Combined DT - walking the same path while carrying a glass of water and performing the described mathematical task.

The order of testing was randomized using a simple PC program. Four walking sequences per one walking condition were recorded for all subjects, with 5 min of rest between two consecutive trials and different walking conditions. All participants were walking using their shoes. All the measurements and testing were performed in the ON state.

The study was performed in a 15-meter long and 3- meter wide hallway. This setup provided participants space and time to walk at a natural pace steadily.

\subsubsection{Instrumentation}

We used the previously described custom-made wireless sensor system (SENSY) that includes two inertial measurement units (IMUs) and two shoe insoles with force sensing resistors (FSR) [20]. Each shoe insole has three FSR sensors positioned below the area of the 2nd and 4th metatarsal/phalanges proximal bones and calcaneus bone, respectively. Shoe insoles were provided in the corresponding size for each subject. IMUs comprise 3D accelerometers and 3D gyroscope sensors. During the measurement process, the shoe insoles were positioned in the subjects' shoe, whereas IMUs were placed laterally on each foot.

\subsubsection{Gait assessment}

The recorded signals were processed and analyzed by custom-made software. The walking sequences were divided into individual walking cycles (i.e., strides) using the recorded FSR signals [21]. The first and last two strides were excluded from further analysis, since initiation and termination of the walking may result in modified gait patterns. The selected strides were described with the following parameters: 1 ) stride length expressed in meters (m);2) stride duration expressed in seconds (s); and 3) swing phase duration expressed in seconds (s). These parameters were calculated using the FSR and IMU data, as previously described [22].

In order to describe gait intra-variability, a relative change of the introduced parameters was calculated as the difference between parameter values of two consecutive strides divided by the parameter value of the previous stride, expressed as a percentage. The relative change of each of the parameters was described with mean value and standard deviation, calculated for each walking sequence and task separately.

\subsection{Statistical analysis}

Continuous variables corresponding to the clinical and gait data were compared between PD-NonDep and PD-Dep groups according to their mean values, using a parametric $t$-test for two independent samples or Mann Whitney non-parametric test. Demographic data were compared using the parametric one-way analysis of variance (ANOVA). The Kolmogorov-Smirnov test was applied to assess the distribution normality of investigated demographic, clinical, and gait parameters.

A linear mixed model was used as a method for analyzing the relationship between potential predictors and dependent variables: the relative change of stride duration, the relative change of stride length, and the relative change of swing time. In this paper, both univariate and multivariate models were considered. Group (PD-Dep, PD-NonDep, and $\mathrm{HC}$ as reference category), Task (Single-task as the reference category, Motor DT, Cognitive DT, Combined DT), Task repetition, Age, HDRS, $\mathrm{FAB}$, and TBS were used as predictors in the univariate models. In contrast, for the multivariate models, only the predictors showing statistical significance were considered. Statistically significant differences were found for values $\mathrm{p}<0.05$. Statistical analysis was performed in SPSS v25.0 (IBM, Armonk, NY, USA).

\section{Results}

\subsection{Criteria for major depression}

Criteria for MD were met by 16 patients, out of which seven were on selective serotonin reuptake inhibitors, two on amitriptyline, two on venlafaxine, and 5 of them on mianserin therapy. All patients with the diagnosis of MD were in a depressive episode during the examination. In the PD-NonDep group, five patients were on SSRIs, although they did 
not meet the criteria for MD.

\subsection{Comparison of clinical features of PD-NonDep and PD-Dep groups}

By considering demographic and clinical characteristics of patients (Table 1), there were no differences in the duration of PD, age between the tested groups, and the motor parts of the UPDRS scores between the PD- Dep and PD-NonDep groups, although the total UPDRS score was significantly higher for patients with depression. The HDRS and TBS scores were significantly different between the patient groups, whereas the FAB scores were not different between PD-Dep and PD- NonDep patients (Table 1). Furthermore, ACE-R, Digit Span Backwards, and Stroop interference scores were not significantly different between PDDep and PD- NonDep groups (Table 1).

In order to examine if cognitive capacity influenced calculation during the DT, the sub-score for calculation task was also analyzed while performing the MMSE test. It was shown that most patients had 4 or 5 points on that task. Only one patient from the PD-Dep group scored with 3 points on the calculation task.

\subsection{Gait parameters}

In this study, 9,266 strides in total were included in the analysis: 3,295 from PD-NonDep patients (773 for the single-task, 825 for motor DT, 832 for cognitive DT, 865 for combined DT), 3,792 from PD-Dep patients ( 870 for the single-task, 883 for motor DT, 973 for cognitive DT, 1,066 for combined DT), and 2,179 from HC subjects (535 for the single-task, 549 for motor DT, 544 for cognitive DT, 551 for combined DT). PD-NonDep and PD-Dep patients walked with an average gait speed of $0.76 \mathrm{~m} / \mathrm{s}$ and $0.81 \mathrm{~m} / \mathrm{s}$, respectively, whereas the average gait speed was $1.27 \mathrm{~m} / \mathrm{s}$ for the HC group. Table 2 presents the results for the relative change of the introduced gait parameters: stride duration, stride length, and swing time. The results are presented for each group, each task, and each task repetition separately.

\subsection{Comparison of gait parameters using linear mixed models}

The univariate linear mixed model analysis showed that variability of stride length was significantly higher in PD- NonDep patients $(\mathrm{p}=0.022)$ and PD-Dep patients $(\mathrm{p}=0.003)$ compared to controls. Similar results were obtained for comparison with patients with higher HDRS scores $(\mathrm{p}=0.044)$, patients with lower FAB and TBS scores ( $\mathrm{p}=0.009$ and $\mathrm{p}<0.001$, respectively), and while performing cognitive and combined DT ( $\mathrm{p}<0.001$ and $\mathrm{p}<0.001$, respectively) compared to

\section{Table 1}

Demographic and clinical characteristics (mean \pm SDs) for three subject groups, together with p-values describing the level of statistically significant differences between the PD-NonDep and PD-Dep groups. ACE-R: Addenbrook's Cognitive Examination-Revised Scale; Dep: Depression; FAB: Frontal Assessment Battery; HC: Healthy controls; HDRS: Hamilton Depression Rating Scale; PD: Parkinson's disease; TBS: Tinetti Balance Score; UPDRS: Unified Parkinson's Disease Rating Scale.

\begin{tabular}{lllll}
\hline Group & $\begin{array}{l}\text { PD-NonDep } \\
(\mathrm{n}=14)\end{array}$ & $\begin{array}{l}\text { PD-Dep } \\
(\mathrm{n}=16)\end{array}$ & $\begin{array}{l}\text { HC } \\
(\mathrm{n}=15)\end{array}$ & $\begin{array}{l}p \\
\text { values }\end{array}$ \\
\hline Age (years) & $65.9 \pm 7.9$ & $63.9 \pm 6.9$ & $62.5 \pm 6.9$ & 0.472 \\
$\quad \begin{array}{l}\text { Duration of the } \\
\quad \text { disease (years) }\end{array}$ & $8.3 \pm 7.6$ & $9.6 \pm 4.2$ & $/$ & 0.588 \\
$\quad$ UPRDS part III & $39.6 \pm 15.2$ & $43.7 \pm 16.6$ & $/$ & \\
HDRS & $6.9 \pm 4.1$ & $19.7 \pm 4.6$ & $/$ & 0.496 \\
FAB & $14.6 \pm 3.2$ & $14.1 \pm 2.8$ & $/$ & $0.000^{*}$ \\
TBS & $22.5 \pm 6.12$ & $17.8 \pm 5.15$ & $/$ & 0.610 \\
ACE-R & $86.8 \pm 8.8$ & $84.7 \pm 8.4$ & $/$ & 0.010 \\
Digit Span & $5.4 \pm 1.6$ & $4.7 \pm 2.2$ & $/$ & 0.512 \\
$\quad$ Backwards & & & & 0.434 \\
$\quad$ Stroop interference & $25.6 \pm 17.5$ & $25.3 \pm 12.2$ & $/$ & 0.956 \\
$\quad$ score & & & & \\
\hline
\end{tabular}

the single-task. The variability of stride length was not significantly different between PD-Dep and PD-NonDep patients. The multivariate linear mixed model with stride length as the dependent variable showed that patients performing cognitive and combined DT $(\mathrm{p}<0.001$ and $\mathrm{p}<0.001$, respectively), and patients with lower TBS $(\mathrm{p}=0.016)$ had higher variability of gait (Table 3 ).

Similarly, the univariate linear mixed model analysis showed that variability of stride duration was significantly higher in PD-NonDep ( $\mathrm{p}=0.002)$ and PD-Dep $(\mathrm{p}=0.001)$ compared to the control group. The stride duration variability was not significantly different between PD-Dep and PD-NonDep patients. A multivariate linear mixed model with stride duration as the dependent variable showed that variability was higher while performing combined and cognitive DT. Stride duration variability is higher in subjects with a lower TBS score $(\mathrm{p}<0.001)$.

Linear mixed model, univariate analysis, showed that swing time variability was statistically significantly higher in PD-Dep patients compared to controls $(\mathrm{p}=0.001)$ and also was higher compared to PDNonDep patients, but this difference was on the boundary of statistical significance $(p=0.063)$. Variability of swing time was significantly higher in patients while performing cognitive and combined tasks ( $\mathrm{p}<0.001$ and $\mathrm{p}<0.001$, respectively) compared to the single-task, and it was significantly lower for task repetition $(\mathrm{p}=0.038)$. Patients with higher swing time variability had significantly higher HDRS scores $(\mathrm{p}=0.01)$ (Fig. 1) and had significantly lower TBS ( $\mathrm{p}=0.005)$. A multivariate linear mixed model with swing time as dependent variable showed that PD-Dep patients had significantly higher variability of gait compared to controls $(p=0.03)$, and while performing cognitive and combined tasks ( $\mathrm{p}<0.001$ and $\mathrm{p}<0.001$, respectively) compared to the single-task but was significantly lower for task repetition $(\mathrm{p}=0.038)$ (Table 4).

The linear mixed models with the variability of stride duration, stride length and swing time as dependent variables within groups showed that the effect of DT cost, or increase in variability during the performance of cognitive and combined DT, was higher in the PD-Dep compared to the PD-NonDep patient group (Table 5).

\section{Discussion}

Our study showed that the variability of gait is associated with depression in PD patients, especially swing time variability, which is possibly one of the most sensitive gait parameters. We also showed that the effect of depression on the variability of gait parameters is more prominent while performing DT. Swing time variability significantly correlated with the depression score, and there was also a difference in swing time variability between the PD-Dep and PD-NonDep group of patients that was close to the level of statistical significance.

Depression is frequently associated with $\mathrm{PD}$, affecting approximately $35 \%$ of patients, and strongly influencing motor and cognitive domains of the disease, as well as health-related quality of life [23]. The mechanisms of depression in PD are complex and traditionally include the participation of dopaminergic, serotonergic, and noradrenergic systems [24]. It has also been found that depressive symptoms in PD were correlated with central cholinergic hypofunction, which has also been identified as an independent risk factor for dementia in PD [25], and linked with gait disorders and postural instability in PD [26]. Also, depression contributed to the variability of stride duration in PD patients, both in ON and OFF time [27]. Hausdorf et al. [28] demonstrated that MD and bipolar disorders contributed to significantly higher variability of swing time, but not to the stride length and stride duration, when compared to controls, suggesting that swing time could be a particularly sensitive marker of subtle changes of the neuronal control of gait or to an impact of depression. Patients with mild PD also had greater swing time variability in comparison to stride duration [29], indicating that in the early course of PD, under conditions of small variability, swing time might be the first parameter to be disrupted in terms of a gait irregularity. In subjects older than 75 years who did not meet criteria for 
Table 2

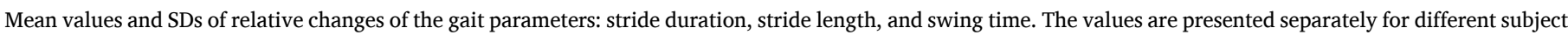
groups, tasks, and task repetitions. Dep: Depression; DT: Dual-task; HC: Healthy controls; PD: Parkinson's disease.

\begin{tabular}{|c|c|c|c|c|c|c|c|c|c|c|c|c|c|}
\hline \multirow{3}{*}{ Task } & \multirow{3}{*}{ Group } & \multicolumn{4}{|c|}{ Stride duration [\%] } & \multicolumn{4}{|c|}{ Stride length [\%] } & \multicolumn{4}{|c|}{ Swing time [\%] } \\
\hline & & \multicolumn{4}{|c|}{ Task repetition } & \multicolumn{4}{|c|}{ Task repetition } & \multicolumn{4}{|c|}{ Task repetition } \\
\hline & & 1 & 2 & 3 & 4 & 1 & 2 & 3 & 4 & 1 & 2 & 3 & 4 \\
\hline \multirow{3}{*}{ Single-task } & PD-Dep & $2.6 \pm 2.3$ & $2.8 \pm 2.7$ & $2.2 \pm 1.8$ & $2.3 \pm 2.2$ & $5.7 \pm 4.5$ & $5.4 \pm 4.3$ & $5.6 \pm 4.8$ & $5.6 \pm 4.8$ & $14.3 \pm 12.9$ & $16.2 \pm 15.1$ & $16.3 \pm 13.9$ & $15.9 \pm 14.8$ \\
\hline & PD-NonDep & $3.1 \pm 3.4$ & $2.4 \pm 2.7$ & $2.7 \pm 3.1$ & $2.5 \pm 2.9$ & $6.3 \pm 6.1$ & $6.1 \pm 9.9$ & $6.6 \pm 6.8$ & $5.7 \pm 5.9$ & $9.8 \pm 9.2$ & $10.1 \pm 9.5$ & $10.4 \pm 9.3$ & $9.2 \pm 8.4$ \\
\hline & $\mathrm{HC}$ & $1.5 \pm 1.4$ & $1.2 \pm 1.2$ & $1.2 \pm 1.1$ & $1.2 \pm 1.0$ & $3.8 \pm 3.1$ & $3.9 \pm 2.8$ & $3.8 \pm 2.9$ & $4.0 \pm 2.8$ & $6.9 \pm 6.0$ & $7.2 \pm 7.3$ & $6.2 \pm 5.6$ & $7.2 \pm 6.2$ \\
\hline & PD-Dep & $2.5 \pm 2.5$ & $2.4 \pm 2.2$ & $2.3 \pm 2.2$ & $2.3 \pm 2.5$ & $5.4 \pm 4.6$ & $5.5 \pm 4.2$ & $5.7 \pm 5.3$ & $5.5 \pm 5.5$ & $15.0 \pm 12.4$ & $15.9 \pm 12.7$ & $15.4 \pm 14.2$ & $13.3 \pm 12.6$ \\
\hline \multirow[t]{3}{*}{ Motor DT } & PD-NonDep & $2.8 \pm 3.0$ & $2.4 \pm 2.9$ & $2.3 \pm 2.0$ & $2.4 \pm 3.0$ & $5.6 \pm 6.0$ & $5.4 \pm 5.4$ & $5.5 \pm 4.4$ & $5.8 \pm 5.5$ & $11.3 \pm 10.9$ & $9.7 \pm 11.5$ & $9.4 \pm 8.4$ & $10.2 \pm 8.5$ \\
\hline & $\mathrm{HC}$ & $1.4 \pm 1.3$ & $1.2 \pm 1.1$ & $1.3 \pm 1.2$ & $1.2 \pm 1.2$ & $4.0 \pm 3.2$ & $3.5 \pm 2.5$ & $4.0 \pm 3.2$ & $3.7 \pm 2.8$ & $6.4 \pm 5.4$ & $6.9 \pm 5.8$ & $5.9 \pm 5.2$ & $6.6 \pm 5.5$ \\
\hline & PD-Dep & $3.8 \pm 3.8$ & $4.0 \pm 5.7$ & $4.7 \pm 5.5$ & $3.7 \pm 3.5$ & $7.9 \pm 7.0$ & $7.8 \pm 7.9$ & $8.2 \pm 8.7$ & $7.4 \pm 7.4$ & $17.8 \pm 19.5$ & $20.0 \pm 18.5$ & $20.8 \pm 20.4$ & $19.2 \pm 26.3$ \\
\hline \multirow[t]{3}{*}{ Cognitive DT } & PD-NonDep & $5.0 \pm 17.3$ & $3.0 \pm 3.3$ & $3.4 \pm 3.9$ & $2.8 \pm 2.5$ & $6.4 \pm 5.8$ & $6.1 \pm 4.7$ & $6.8 \pm 6.7$ & $6.4 \pm 5.5$ & $11.5 \pm 10.6$ & $11.3 \pm 10.2$ & $9.7 \pm 10.1$ & $10.3 \pm 9.3$ \\
\hline & $\mathrm{HC}$ & $2.0 \pm 1.9$ & $1.6 \pm 1.6$ & $1.3 \pm 1.1$ & $1.6 \pm 1.6$ & $4.9 \pm 3.7$ & $4.7 \pm 3.5$ & $4.1 \pm 3.3$ & $4.5 \pm 3.4$ & $6.4 \pm 4.8$ & $6.8 \pm 5.2$ & $6.8 \pm 5.4$ & $7.2 \pm 5.3$ \\
\hline & PD-Dep & $3.8 \pm 3.5$ & $3.3 \pm 3.3$ & $3.8 \pm 3.8$ & $3.2 \pm 3.1$ & $7.0 \pm 7.0$ & $7.9 \pm 7.1$ & $8.3 \pm 10.7$ & $7.6 \pm 7.3$ & $19.1 \pm 19.7$ & $20.3 \pm 19.4$ & $20.3 \pm 20.7$ & $15.6 \pm 13.8$ \\
\hline \multirow[t]{2}{*}{ Combined DT } & PD-NonDep & $3.6 \pm 4.8$ & $3.3 \pm 4.7$ & $3.7 \pm 4.1$ & $3.2 \pm 3.2$ & $6.8 \pm 7.6$ & $6.0 \pm 5.9$ & $5.6 \pm 5.8$ & $6.2 \pm 5.5$ & $11.8 \pm 10.7$ & $11.6 \pm 10.9$ & $11.5 \pm 10.5$ & $11.5 \pm 12.2$ \\
\hline & $\mathrm{HC}$ & $1.7 \pm 1.6$ & $1.6 \pm 1.4$ & $1.6 \pm 1.4$ & $1.6 \pm 1.5$ & $4.6 \pm 3.6$ & $4.2 \pm 3.4$ & $4.1 \pm 3.3$ & $4.6 \pm 3.3$ & $6.5 \pm 5.1$ & $7.0 \pm 5.8$ & $7.0 \pm 6.1$ & $7.0 \pm 5.6$ \\
\hline
\end{tabular}

Table 3

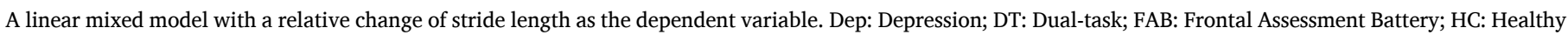
controls; HDRS: Hamilton Depression Rating Scale; PD: Parkinson's disease; TBS: Tinetti Balance Score.

\begin{tabular}{|c|c|c|c|c|c|c|}
\hline Group & $\mathrm{B}$ & SE & p. & B & SE & p. \\
\hline PD-Dep & 2.49 & 0.78 & 0.003 & 0.52 & 1.01 & 0.607 \\
\hline PD-NonDep & 1.89 & 0.80 & 0.022 & 0.67 & 0.87 & 0.446 \\
\hline $\mathrm{HC}$ & Reference & Category & & Reference & Category & \\
\hline \multicolumn{7}{|l|}{ Task } \\
\hline Single-task & Reference & Category & & Reference & Category & \\
\hline Motor DT & -0.24 & 0.14 & 0.084 & -0.24 & 0.14 & 0.084 \\
\hline Cognitive DT & 1.04 & 0.14 & $<0.001$ & 1.04 & 0.14 & $<0.001$ \\
\hline Combined DT & 0.88 & 0.14 & $<0.001$ & 0.88 & 0.14 & $<0.001$ \\
\hline Task repetition & -0.03 & 0.05 & 0.525 & & & \\
\hline Age & 0.06 & 0.05 & 0.200 & & & \\
\hline HDRS & 0.09 & 0.04 & 0.044 & & & \\
\hline FAB & -0.31 & 0.11 & 0.009 & -0.11 & 0.12 & 0.366 \\
\hline TBS & -0.37 & 0.09 & $<0.001$ & -0.29 & 0.11 & 0.016 \\
\hline
\end{tabular}

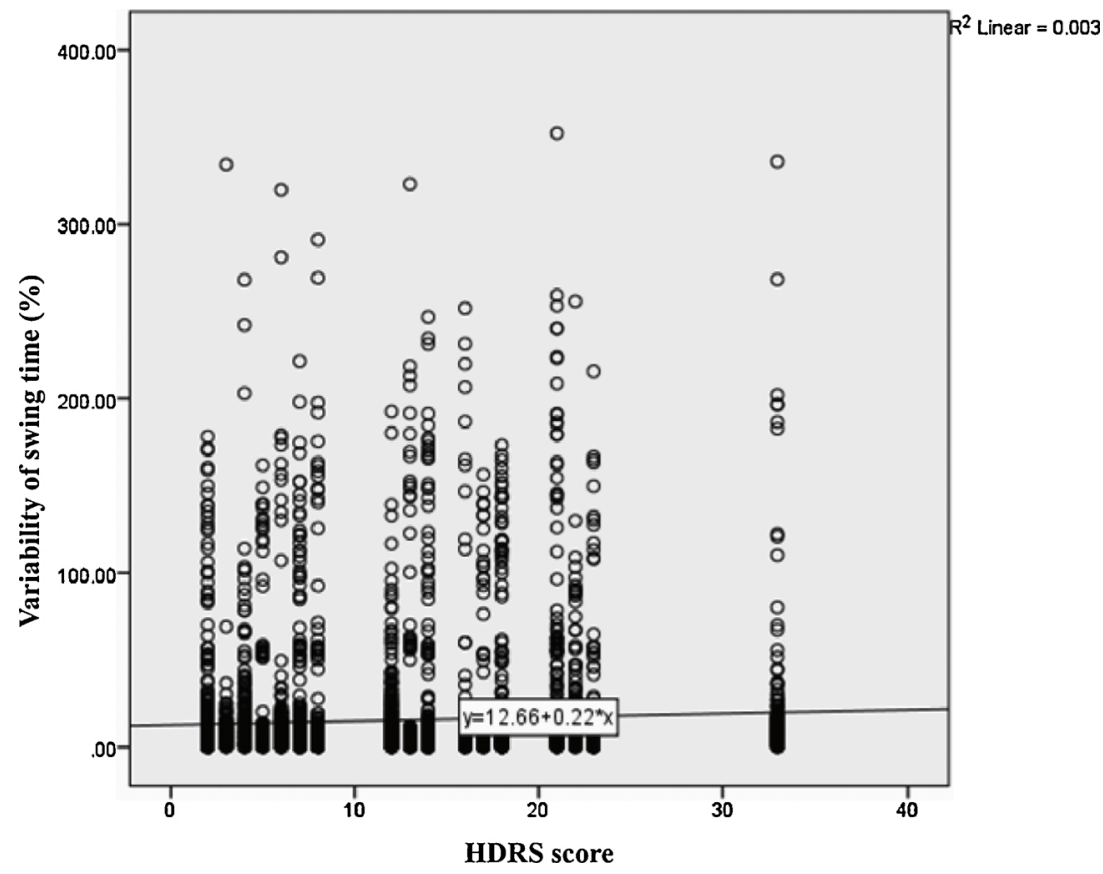

Fig. 1. Correlation between the variability of swing time and the HDRS score. HDRS: Hamilton Depression Rating Scale. 
Table 4

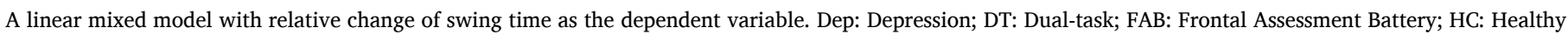
controls; HDRS: Hamilton Depression Rating Scale; PD: Parkinson's disease; TBS: Tinetti Balance Score.

\begin{tabular}{|c|c|c|c|c|c|c|}
\hline Group & $\mathrm{B}$ & SE & p. & B & SE & p. \\
\hline PD-Dep & 10.14 & 2.72 & 0.001 & 7.99 & 3.56 & 0.030 \\
\hline PD-NonDep & 3.70 & 2.77 & 0.190 & 2.51 & 3.04 & 0.412 \\
\hline $\mathrm{HC}$ & Reference & Category & & Reference & Category & \\
\hline \multicolumn{7}{|l|}{ Task } \\
\hline Single-task & Reference & Category & & Reference & Category & \\
\hline Motor DT & -0.29 & 0.26 & 0.256 & -0.29 & 0.26 & 0.256 \\
\hline Cognitive DT & 1.51 & 0.26 & $<0.001$ & 1.51 & 0.26 & $<0.001$ \\
\hline Combined DT & 1.63 & 0.26 & $<0.001$ & 1.63 & 0.26 & $<0.001$ \\
\hline Task repetition & -0.17 & 0.08 & 0.038 & -0.17 & 0.08 & 0.038 \\
\hline Age & -0.21 & 0.18 & 0.237 & & & \\
\hline HDRS & 0.41 & 0.15 & 0.010 & & & \\
\hline FAB & -0.45 & 0.44 & 0.307 & & & \\
\hline TBS & -1.01 & 0.34 & 0.005 & -0.40 & 0.43 & 0.359 \\
\hline
\end{tabular}

Table 5

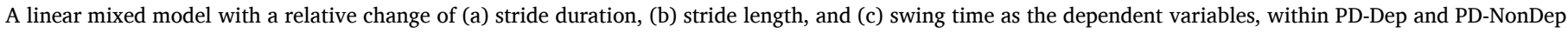
groups. Dep: Depression; DT: Dual-task; PD: Parkinson's disease.

\begin{tabular}{|c|c|c|c|c|c|c|}
\hline \multirow{2}{*}{$\begin{array}{l}\text { Task } \\
\text { stride duration }\end{array}$} & \multicolumn{3}{|l|}{ PD-Dep } & \multicolumn{3}{|c|}{ PD-NonDep } \\
\hline & B & SE & p. & B & SE & p. \\
\hline Single-task & Reference & Category & & Reference & Category & \\
\hline Motor DT & -0.1 & 0.15 & 0.497 & -0.18 & 0.25 & 0.474 \\
\hline Cognitive DT & 1.56 & 0.15 & $<0.001$ & 0.87 & 0.25 & 0.001 \\
\hline Combined DT & 1.06 & 0.15 & $<0.001$ & 0.79 & 0.25 & 0.002 \\
\hline \multicolumn{7}{|l|}{ stride length } \\
\hline Single-task & Reference & Category & & Reference & Category & \\
\hline Motor DT & -0.03 & 0.29 & 0.905 & -0.63 & 0.27 & 0.021 \\
\hline Cognitive DT & 2.24 & 0.29 & $<0.001$ & 0.22 & 0.27 & 0.424 \\
\hline Combined DT & 2.15 & 0.29 & $<0.001$ & -0.04 & 0.27 & 0.880 \\
\hline \multicolumn{7}{|l|}{ swing time } \\
\hline Single-task & Reference & Category & & Reference & Category & \\
\hline Motor DT & -0.74 & 0.60 & 0.216 & 0.3 & 0.44 & 0.487 \\
\hline Cognitive DT & 3.78 & 0.60 & $<0.001$ & 0.84 & 0.44 & 0.053 \\
\hline Combined DT & 3.2 & 0.60 & $<0.001$ & 1.74 & 0.44 & $<0.001$ \\
\hline
\end{tabular}

MD, it has been shown that contrary to stride duration and stride length, swing time variability was clearly associated with symptoms of depression, even when factors such as vascular comorbidity and cognitive status were taken into account [30]. Moreover, Frenkel-Toledo et al. [31] have demonstrated that contrary to stride duration variability, swing time variability was independent of gait speed, which implied possible different mechanisms for these two gait parameters. Although we could not confirm that depression was an independent factor for the variability of three gait parameters, probably due to the small number of patients, PD-Dep group of patients had significantly higher swing time variability compared to control group while this was not shown with PD-NonDep patients. HDRS score was not just significantly correlated with the swing time variability but it was significantly higher in patients with higher stride length variability.

The motor-cognitive DT paradigm has been used to examine an interplay between gait and cognition [32,33], affecting gait variability both in healthy subjects and PD patients [3,34], with consequent increase of the risk of falls and worsened daily functioning [4]. Even in cognitively normal, older adults, there was a robust association of gait speed and gait variability with executive function, attention, and (to a lesser extent) memory and visuospatial function, that was more emphatic under DT conditions [33,35-38]. Attention and executive functions were impaired early in PD and were manifested in an inability for adequate separation of cognitive resources required for the simultaneous performance of activities during DT [5,39]. Similar to our results, it has been shown that DT affected the swing time and step length variability, as well as double support time in $\mathrm{PD}$, particularly in those with mild cognitive impairment when compared to the control group [40]. Patients with MD performed the Walking Trial Making Test more slowly, with lower gait accuracy and higher DT costs when compared to controls [41]. Late-onset depression in subjects without PD has been coupled with cognitive disturbances and, in particular, with executive dysfunction, probably reflecting shared frontostriatal pathology [42]. Results on executive function tests were a predictive factor for higher variability of gait parameters when performing DT in older patients with MD who were under the risk of falls [41]. Gabel et al. [43] found that older patients with MD did not exhibit differences in the variability of gait parameters when compared to non-depressive matched subjects, either during single- task conditions or during DT performance. However, results of cognitive testing indicated a link with increased variability during DT performance only in the group of depressed, but not in the group of non-depressed patients. DT significantly increased the variability of all three gait parameters in our study, and performing DT was an independent factor for variability when using all three parameters as the dependent variable. Besides this, the variability of stride length was higher in patients with the higher FAB score but not variability of swing time, and FAB did not turn out to be an independent variable. Also, there was no difference in the FAB, ACE-R, Digit Span Backwards and Stroop inference scores between PD-Dep and PD- NonDep patients, which, together with the results for the HDRS score, implied that the influence of depression is partially independent of executive functions. Although DT significantly affected the variability of all three parameters, this effect was more expressed in the group of depressed patients meaning that depression probably influenced gait 
parameters, especially in conditions of additional cognitive or combined tasks. Nevertheless, it is possible that DT performance detects more subtle cognitive decrements than those observed in the used executive tests.

Variability of three gait parameters was more expressed in patients who had lower TBS scores, and TBS was an independent predictor for swing time variability. Depression represents an independent risk factor for falls in older individuals, where several studies have demonstrated a connection between depression and postural instability [44]. Previous studies have demonstrated that older patients with major depression, who are under the risk of falling, have lower scores on executive function tests, which had predictive significance for gait variability under DT conditions compared to base gait [43]. They proposed the hypothesis that executive functions contribute to greater problems in walking for older individuals with depression compared to those without depression, but at the same time, no difference was found in gait parameter variability under DT conditions compared to base conditions. In our study, patients with depression and those without depression had significantly different TBS scores, although there was no significant difference in executive functions in the FAB test. PD patients also exhibit a change in this nucleus [45]. Moreover, observed changes in cholinergic function in patients with PD and depression and demonstrated the influence of cholinergic dysfunction on gait and postural control could point to a similar pathophysiological origin. Depression is coupled with axial motor symptoms in PD and appears more frequently in a form with pronounced postural instability and gait disorders [46,47]. A recent study has demonstrated that patients with PD and comorbid depression exhibit differences in postural control compared to patients without depression, wherein the upright position depressive patients exhibit greater flexion in the pelvic area, causing leaning forward of the torso [48]. In our study, patients with PD and depression had significantly lower scores on the TBS, which suggests that depression could additionally impair balance and contribute to falls.

The main limitation of our study was a relatively small number of patients. However, we performed a detailed clinical and psychological evaluation. The diagnosis of MD was based on standardized psychiatric instruments, and not just by using depression scales. Furthermore, gait was tested using wireless sensors (closer to normal gait conditions than testing on a treadmill).

The clinical implication of the possible influence of depression on gait parameters is important, especially the variability of gait since it is well known that the variability of gait is one of the main risk factors for falls. Our results confirmed that recognizing depression and its treatment could be one of the possible strategies for improving balance and gait. In conclusion, we have demonstrated that MD in PD was associated with gait variability, particularly during DT conditions, which has been previously shown to be a significant predictor of future falls. Therefore, strategies for improvement of gait and reduction of fall risks in PD may include clinical efforts for early recognition and treatment of depression.

Future studies should include a greater number of patients, including those that meet minor depression criteria, as well as subsyndromal depression (patients who experience depression only in OFF periods). It would also be interesting to test, on a larger group of patients whether there are differences in gait parameters in patients who have diagnosed major depression but have emerged from an episode and are taking adequate therapy, and to test how the DT paradigm affects patients with depression, but without PD.

\section{Funding}

This research was in part funded by the Serbian Ministry of Education, Science and Technological Development [grant number OI175090 and OI-175016].

\section{CRediT authorship contribution statement}

Nataša T Dragašević- Mišković: Conceptualization, Methodology, Investigation, Writing - original draft. Vladislava Bobić: Investigation, Methodology, Formal analysis, Writing - original draft. Milutin Kostić: Methodology, Investigation. Iva Stanković: Methodology, Investigation. Saša Radovanović: Conceptualization, Methodology, Investigation, Writing - review \& editing. Kosta Dimitrijević: Methodology, Investigation. Marina Svetel: Methodology, Investigation. Igor Petrović: Conceptualization, Investigation. Milica Đurić- Jovičić: Conceptualization, Formal analysis, Writing - review \& editing, Supervision.

\section{Declaration of Competing Interest}

None.

\section{Acknowledgment}

We would like to thank prof. Goran Trajković for his help in the methodological approach to a statistical assessment of our results.

\section{References}

[1] D.S. Peterson, F.B. Horak, The effect of levodopa on improvements in protective stepping in people with Parkinson's disease, Neurorehabil. Neural Repair 30 (2016) 931-940, https://doi.org/10.1177/1545968316648669.

[2] G. Yogev- Seligmann, J.M. Hausdorff, N. Giladi, The role of executive function and attention in gait, Mov. Disord. 23 (2008) 329-342, https://doi.org/10.1002/ mds. 21720 .

[3] G. Yogev, N. Giladi, C. Peretz, S. Springer, E.S. Simon, J.M. Hausdorff, Dual tasking, gait rhythmicity, and Parkinson's disease: Which aspects of gait are attention demanding? Eur. J. Neurosci. 22 (2005) 1248-1256, https://doi.org/ 10.1111/j.1460-9568.2005.04298.x.

[4] M. Plotnik, N. Giladi, Y. Dagan, J.M. Hausdorff, Postural instability and fall risk in Parkinson's disease: impaired dual tasking, pacing, and bilateral coordination of gait during the "ON" medication state, Exp. Brain Res. (2011) 529-538, https:// doi.org/10.1007/s00221-011-2551-0. Springer.

[5] L. Rochester, A. Nieuwboer, K. Baker, V. Hetherington, A.-M. Willems, G. Kwakkel, E. Van Wegen, I. Lim, D. Jones, Walking speed during single and dual tasks in Parkinson's disease: Which characteristics are important? Mov. Disord. 23 (2008) 2312-2318, https://doi.org/10.1002/mds.22219.

[6] J.S.A.M. Reijnders, U. Ehrt, W.E.J. Weber, D. Aarsland, A.F.G. Leentjens, A systematic review of prevalence studies of depression in Parkinson's disease, Mov. Disord. 23 (2008) 183-189, https://doi.org/10.1002/mds.21803.

[7] A.I. Tröster, L.D. Stalp, A.M. Paolo, J.A. Fields, W.C. Koller, Neuropsychological impairment in Parkinson's disease with and without depression, Arch. Neurol. 52 (1995) 1164-1169, https://doi.org/10.1001/archneur.1995.00540360042014.

[8] M.R. Lemke, T. Wendorff, B. Mieth, K. Buhl, M. Linnemann, Spatiotemporal gait patterns during over ground locomotion in major depression compared with healthy controls, J. Psychiatr. Res. 34 (2000) 277-283, https://doi.org/10.1016/ S0022-3956(00)00017-0.

[9] L. Sloman, M. Berridge, S. Homatidis, D. Hunter, T. Duck, Gait patterns of depressed patients and normal subjects, Am. J. Psychiatry 139 (1982) 94-97, https://doi.org/10.1176/ajp.139.1.94.

[10] A.J. Hughes, S.E. Daniel, L. Kilford, A.J. Lees, Accuracy of clinical diagnosis of idiopathic Parkinson's disease: a clinico-pathological study of 100 cases, J. Neurol. Neurosurg. Psychiatry 55 (1992) 181-184, https://doi.org/10.1136/ jnnp.55.3.181.

[11] R.M. of the U.D.C. Fahn, S. Elton, The unified Parkinson's disease rating scale, Recent Dev. Park. Dis. 2 (1987) 293-304, 153-163.

[12] M. First, J. Williams, R. Karg, R. Spitzer-Arlington, Structured clinical interview for DSM- 5R-research version (SCID-5 for DSM-5 research version; SCID-5-RV) Arlington, VA Am. Psychiatr. Assoc. (2015) 1-94.

[13] M. Hamilton, A rating scale for depression, J. Neurol. Neurosurg. Psychiatry. 23 (1960) 56-62, https://doi.org/10.1136/jnnp.23.1.56.

[14] B. Dubois, A. Slachevsky, I. Litvan, B. Pillon, The FAB: A frontal assessment battery at bedside, Neurology 55 (2000) 1621-1626, https://doi.org/10.1212/ WNL.55.11.1621.

[15] E. Mioshi, K. Dawson, J. Mitchell, R. Arnold, J.R. Hodges, The Addenbrooke's Cognitive Examination revised (ACE-R): a brief cognitive test battery for dementia screening, Int. J. Geriatr. Psychiatry 21 (2006) 1078-1085, https://doi.org/ 10.1002/gps.1610.

[16] D. Wechsler, Wechsler memory scale -revised: manual, Psychol. Corp. (1987).

[17] C.J. Golden, A group version of the Stroop Color and Word Test, J. Pers. Assess. 39 (1975) 386-388, https://doi.org/10.1207/s15327752jpa3904_10.

[18] M.F. Folstein, S.E. Folstein, P.R. McHugh, "Mini-mental state" a practical method for grading the cognitive state of patients for the clinician, J. Gsychiaf. Res. 12 (1975) 189-198. 
[19] S. Radovanović, M. Jovičić, N.P. Marić, V. Kostić, Gait characteristics in patients with major depression performing cognitive and motor tasks while walking, Psychiatry Res. 217 (2014) 39-46, https://doi.org/10.1016/j. psychres.2014.02.001.

[20] M.D. Djurić- Jovičić, N.S. Jovičić, D.B. Popović, A.R. Djordjević, Nonlinear optimization for drift removal in estimation of gait kinematics based on accelerometers, J. Biomech. 45 (2012) 2849-2854, https://doi.org/10.1016/j. jbiomech.2012.08.028.

[21] V.N. Bobic, M.D. Djuric-Jovicic, S.M. Radovanovic, N.T. Dragasevic, V.S. Kostic, M. B. Popovic, Challenges of stride segmentation and their implementation for impaired gait, in: Proc. Annu. Int. Conf. IEEE Eng. Med. Biol. Soc. EMBS, Institute of Electrical and Electronics Engineers Inc., 2018, pp. 2284-2287, https://doi.org/ 10.1109/EMBC. 2018.8512836.

[22] M.D. Djurić- Jovičić, N.S. Jovičić, S.M. Radovanović, N.D. Kresojević, V.S. Kostić, M.B. Popović, Quantitative and qualitative gait assessments in Parkinson's disease patients, Vojnosanit. Pregl. 71 (2014) 809-816, https://doi.org/10.2298/ VSP130422012D.

[23] R.G. Brown, S. Landau, J.V. Hindle, J. Playfer, M. Samuel, K.C. Wilson, C.S. Hurt, R.J. Anderson, J. Carnell, L. Dickinson, G. Gibson, R. Van Schaick, K. Sellwood, B. A. Thomas, D.J. Burn, Depression and anxiety related subtypes in Parkinson's disease, J. Neurol. Neurosurg. Psychiatry. 82 (2011) 803-809, https://doi.org/ 10.1136/jnnp. 2010.213652.

[24] L. Ishihara, C. Brayne, A systematic review of depression and mental illness preceding Parkinson's disease, Acta Neurol. Scand. 113 (2006) 211-220, https:// doi.org/10.1111/j.1600-0404.2006.00579.x.

[25] N.I. Bohnen, R.L. Albin, The cholinergic system and Parkinson disease, Behav. Brain Res. 221 (2011) 564-573, https://doi.org/10.1016/j.bbr.2009.12.048.

[26] P. Remy, M. Doder, A. Lees, N. Turjanski, D. Brooks, Depression in Parkinson's disease: loss of dopamine and noradrenaline innervation in the limbic system, Brain 128 (2005) 1314-1322.

[27] N. Paker, D. Bugdayci, G. Goksenoglu, D.T. Demircioğlu, N. Kesiktas, N. Ince, Gait speed and related factors in Parkinson's disease, J. Phys. Ther. Sci. 27 (2015) 3675-3679, https://doi.org/10.1589/jpts.27.3675.

[28] J.M. Hausdorff, C.K. Peng, A.L. Goldberger, A.L. Stoll, Gait unsteadiness and fall risk in two affective disorders: a preliminary study, BMC Psychiatry 4 (2004) 1-7, https://doi.org/10.1186/1471-244X-4-39.

[29] R. Baltadjieva, N. Giladi, L. Gruendlinger, C. Peretz, J.M. Hausdorff, Marked alterations in the gait timing and rhythmicity of patients with de novo Parkinson's disease, Eur. J. Neurosci. 24 (2006) 1815-1820, https://doi.org/10.1111/j.14609568.2006.05033.x.

[30] T.C. Brandler, C. Wang, M. Oh-Park, R. Holtzer, J. Verghese, Depressive symptoms and gait dysfunction in the elderly, Am. J. Geriatr. Psychiatry 20 (2012) 425-432, https://doi.org/10.1097/JGP.0b013e31821181c6.

[31] S. Frenkel- Toledo, N. Giladi, C. Peretz, T. Herman, L. Gruendlinger, J. M. Hausdorff, Effect of gait speed on gait rhythmicity in Parkinson's disease: variability of stride time and swing time respond differently, J. Neuroeng. Rehabil. 2 (2005) 23, https://doi.org/10.1186/1743-0003-2-23.

[32] S. Springer, N. Giladi, C. Peretz, G. Yogev, E.S. Simon, J.M. Hausdorff, Dual-tasking effects on gait variability: the role of aging, falls, and executive function, Mov. Disord. 21 (2006) 950-957, https://doi.org/10.1002/mds.20848.
[33] N.E. Fritz, F.M. Cheek, D.S. Nichols-Larsen, Motor-cognitive dual-task training in persons with neurologic disorders: a systematic Rreview, J. Neurol. Phys. Ther. 39 (2015) 142-153, https://doi.org/10.1097/NPT.0000000000000090.

[34] M. Amboni, P. Barone, J.M. Hausdorff, Cognitive contributions to gait and falls: evidence and implications, Mov. Disord. 28 (2013) 1520-1533, https://doi.org/ 10.1002/mds.25674.

[35] K. Marder, M.X. Tang, L. Cote, Y. Stern, R. Mayeux, The frequency and associated risk factors for dementia in patients with parkinson's disease, Arch. Neurol. 52 (1995) 695-701, https://doi.org/10.1001/archneur.1995.00540310069018.

[36] S. Lord, B. Galna, J. Verghese, S. Coleman, D. Burn and, L. Rochester, Independent domains of gait in older adults and associated motor and nonmotor attributes: validation of a factor analysis approach, J. Gerontol. Ser. A 68 (2013) 820-827.

[37] K.L. Martin, L. Blizzard, A.G. Wood, V. Srikanth, R. Thomson, L.M. Sander, M. L. Callisaya, Cognitive function, gait, and gait variability in older people: a population-based study, J. Gerontol. Ser. A 68 (2012) 726-732.

[38] J. Verghese, C. Wang, R.B. Lipton, R. Holtzer, X. Xue, Quantitative gait dysfunction and risk of cognitive decline and dementia, J. Neurol. Neurosurg. Psychiatry 78 (2007) 929-935, https://doi.org/10.1136/jnnp.2006.106914.

[39] J.M. Hausdorff, J. Balash, N. Giladi, Effects of cognitive challenge on gait variability in patients with Parkinson's disease, J. Geriatr. Psychiatry Neurol. 16 (2003) 53-58, https://doi.org/10.1177/0891988702250580.

[40] P.L. Sheridan, J. Solomont, N. Kowall, J.M. Hausdorff, Influence of executive function on locomotor function: divided attention increases gait variability in Alzheimer's disease, J. Am. Geriatr. Soc. 51 (2003) 1633-1637, https://doi.org/ 10.1046/j.1532-5415.2003.51516.x.

[41] S.L. Wright, R.E. Kay, E.T. Avery, B. Giordani, N.B. Alexander, The impact of depression on dual tasking among patients with high fall risk, J. Geriatr. Psychiatry Neurol. 24 (2011) 142-150, https://doi.org/10.1177/0891988711409408.

[42] K.Z.H. Li, L. Bherer, A. Mirelman, I. Maidan, J.M. Hausdorff, Cognitive involvement in balance, gait and dual-tasking in aging: a focused review from a neuroscience of aging perspective, Front. Neurol. 9 (2018) 913, https://doi.org/ 10.3389/fneur.2018.00913.

[43] N.M. Gabel, N.A. Crane, E.T. Avery, R.E. Kay, A. Laurent, B. Giordani, N. B. Alexander, S.L. Weisenbach, Dual-tasking gait variability and cognition in latelife depression, Int. J. Geriatr. Psychiatry 30 (2015) 1120-1128, https://doi.org/ 10.1002/gps.4340.

[44] E. Franzén, D. Conradsson, M. Hagströmer, M.H. Nilsson, Depressive symptoms associated with concerns about falling in Parkinson's disease, Brain Behav. 6 (2016) e00524, https://doi.org/10.1002/brb3.524.

[45] Y. Vermeiren, P.P. De Deyn, Targeting the norepinephrinergic system in Parkinson's disease and related disorders: The locus coeruleus story, Neurochem. Int. 102 (2017) 22-32, https://doi.org/10.1016/j.neuint.2016.11.009.

[46] L. Kadastik-Eerme, M. Muldmaa, S. Lilles, M. Rosenthal, N. Taba, P. Taba, Nonmotor features in Parkinson's disease: what are the most important associated factors? Parkinsons Dis. (2016).

[47] R. Šumec, I. Rektorová, R. Jech, K. Menšíková, J. Roth, E. Růžička, D. Sochorová, L. Dušek, P. Kaňovský, I. Rektor, T. Pavlík, P. Filip, M. Bareš, Motion and emotion: anxiety-axial connections in Parkinson's disease, J. Neural Transm. 124 (2017) 369-377, https://doi.org/10.1007/s00702-016-1652-0.

[48] Y. Kim, S.M. Cheon, C. Youm, M. Son, J.W. Kim, Depression and posture in patients with Parkinson's disease, Gait Posture 61 (2018) 81-85, https://doi.org/10.1016/ j.gaitpost.2017.12.026. 